\title{
Post-chemotherapy retroperitoneal lymph node dissection for non-seminomatous germ cell tumors: A single-surgeon, Canadian experience
}

\author{
Joshua King ${ }^{1}$; Jun Kawakami ${ }^{2}$; Daniel Heng ${ }^{3}$; Chun Loo Gan ${ }^{3}$ \\ ${ }^{1}$ Biological Sciences, University of Lethbridge, Lethbridge, AB, Canada; ${ }^{2}$ Division of Urology, Department of \\ Surgery, Tom Baker Cancer Centre, University of Calgary, Calgary, AB, Canada; ${ }^{3}$ Division of Medical \\ Oncology, Department of Oncology, Tom Baker Cancer Centre, University of Calgary, Calgary, AB, Canada
}

Cite as: Can Urol Assoc J 2020 March 30; Epub ahead of print. http://dx.doi.org/10.5489/cuaj.6219

Published online March 30, 2020

$* * *$

\section{Abstract}

Introduction: Post-chemotherapy retroperitoneal lymph node dissection (PCRPLND) has a well-established role in the management of residual retroperitoneal masses $>1 \mathrm{~cm}$ in patients with advanced non-seminomatous germ cell tumor (NSGCT). Herein, we report our singlesurgeon surgical experience in a Canadian tertiary hospital.

Methods: We identified 57 patients with NSGCT who received primary chemotherapy and PCRPLND from 2010-2016. Surgical complication rate was graded with Clavien-Dindo classification. Chi-squared testing was used in testing for differences in proportion of PCRPLND tumor histology vs. the historical cohorts. Chi-squared testing was also used to analyze the association between primary orchiectomy tumor histology and postchemotherapy residual mass (PCRM) tumor histology.

Results: The overall complication rate was $23 \%(n=13)$, of which four were Clavien-Dindo grade IIIb and one was grade IVa. Fourteen percent of patients required additional procedure for resection of adjacent organs intraoperatively. There was a statistically significant difference in the distribution of PCRPLND tumor histologies (chi-squared $p=0.0187$ ), with a lower rate of viable tumor $(7 \%)$ and higher rate of teratoma (63\%) compared to historical cohorts. The absence of teratoma in the primary orchiectomy specimen was associated with the findings of fibrotic/necrotic tissue in the PCRM (chi-squared $\mathrm{p}=0.0005$ ).

Conclusions: Our series demonstrated that the rate of viable tumor in PCRM appears lower than published historical series, and this possibly reflects the improvement in chemotherapy delivery in a contemporary series. The high rate of teratoma in the PCRM calls for ongoing need for PCRPLND. Grade III and IV surgical complications are considered rare in our series. 


\section{Introduction}

Testicular cancers are the most common solid tumour malignancies of young adult men. In Canada the projected incidence of testicular cancers was estimated to be 1100 cases in 2017 , including 45 deaths. The age-standardized incidence rate was estimated at 6.1/100,000 males with a 5-year survival rate of $96 \%{ }^{1}$

Germ cell tumours (GCTs) comprise 95\% of malignant tumours arising in the testes. GCTs are broadly divided into seminoma and nonseminoma because they have different prognostic and treatment algorithms.

The 1997 International Germ Cell Cancer Collaborative Group (IGCCCG) classification stratify patients with advanced GCTs by risk: good ( $91 \% 5$ year OS rate), intermediate ( $75-83 \% 5$ year OS rate), and poor risk (42-54\% 5 year OS rate). ${ }^{2}$ In 2006, a pooled analysis of chemotherapy trials reported survival in the good-, intermediate-, and poor risk groups to be $94 \%, 83 \%$, and $71 \%$, respectively. ${ }^{3}$ Outcomes were improved compared with the 1997 analysis. ${ }^{3}$

The use of retroperitoneal lymph node dissection (RPLND) in the management of GCTs has evolved over the years, especially since the discovery of highly efficacious chemotherapeutic agents such as cisplatin in 1970s. Principally, there is a shift away from the use of primary RPLNDs in the management of early stage GCTs, mainly due to the widespread uptake of active surveillance as the management strategies in stage I disease and use of induction chemotherapy in stage II disease. ${ }^{4,5}$ The majority of RPLNDs today are performed in the post chemotherapy setting. ${ }^{6}$

Post-chemotherapy retroperitoneal lymph node dissection (PCRPLND) has a wellestablished role in the management of residual masses $>1 \mathrm{~cm}$ in patients with advanced NSGCT with negative serum tumour markers. ${ }^{7}$ The main rationale for PCRPLND is that residual masses $>1 \mathrm{~cm}$ in advanced Non-Seminomatous Germ Cell Tumours (NSGCT) have a $50-70 \%$ chance of harbouring viable tumours or teratoma. Historically, histopathologic evaluation of resected specimens shows necrosis, teratoma and viable malignancy (with or without teratoma) in $40 \%, 45 \%$ and $15 \%$ of cases respectively. ${ }^{8}$ Each of the histopathology findings is of prognostic value and guides further management. Teratoma is known to be chemoresistant, and it has the potential for malignant transformation and late relapse, ${ }^{9}$ and PCRPLND is often curative in this setting. Viable tumours may prompt intervention with salvage chemotherapy. There are many attempts at identifying predictive factors for viable tumours or teratoma at surgery, ${ }^{10}$ but the utility of various predictive models is not widely adopted.

As the only centre for RPLND surgery in Southern Alberta, Canada, we performed a retrospective data collection and analysis of patients who had undergone an open RPLND between 2010 and 2016. Our objective is to present our surgical experience and to evaluate the histopathology from the PCRPLNDs performed at our institution. 


\section{Methods}

The electronic medical records of a single surgeon at the Southern Alberta Institute of Urology (SAIU) were searched for any patients who had undergone an open RPLND for advanced GCTs between 2010 and 2016.

\section{Inclusion criteria}

All patients have either NSGCTs on histology or elevation of serum alpha-feto protein (AFP). They must have received primary chemotherapy prior to surgery and had post chemotherapy retroperitoneal masses greater than $1 \mathrm{~cm}$ in short axis. Patients who received salvage chemotherapy prior to surgery were excluded which aligned with previous published surgical series. ${ }^{9,10,11,12}$ Data were collected by review of electronic medical records, pre-op tumour markers results, radiology reports, operative findings and histopathology report.

\section{Data collection}

Patient's demographic data were collected. Histology of the orchiectomies and resected retroperitoneal specimens were collected from the pathology reports. Patients were stratified into good, intermediate, or poor risk groups based on the IGCCCG classification. Data on the type of chemotherapy regimens used and number of cycles of chemotherapy delivered were also collected. Complications of surgery were defined as any documented surgical complication in the operative report or clinical notes. Intraoperative blood loss was included in intraoperative complications. The severity of complications was graded according to the Clavien-Dindo classification of surgical complications, which ranges from grade 1-5. ${ }^{13}$ Additional procedures were defined as any additional surgical procedure that occurred simultaneously during the PCRPLND and this information was collected.

The histology of post-chemotherapy residual mass (PCRM) was recorded and a chi square test was used to test for the statistical significance of the occurrence of observed histology against the reported histology proportion ( $40 \%$ necrosis, $45 \%$ teratoma and $15 \%$ viable tumours) according to prior published series. Chi square testing was used in testing for differences in proportion of post RPLND tumour histology in this cohort versus the most comprehensive cohort available. ${ }^{8}$ Chi square testing was also used in evaluating the association between the primary tumour histology and the histological findings at PCRM.

\section{Results}

A total of 64 patients were identified. Of these, 7 patients were excluded as 4 patients underwent primary RPLND, 1 patient was considered a desperation case, and 2 patients have pure seminoma histology in the primary tumour. Therefore, a total of 57 patients with advanced NSGCTs who had received primary chemotherapy prior to RPLND met the inclusion criteria for the study. All patients underwent radical orchiectomy. The baseline characteristics are outlined in table 1. Median follow up is 52.4 months. 
The median age of patients is 29 . Overall, the majority of our patients are of IGCCCG good risk $n=32(56 \%)$. The number of patients who were intermediate or poor risk are $n=14(25 \%)$ and $\mathrm{n}=11(19 \%)$, respectively.

The most common ( $\mathrm{n}=47,83 \%$ ) primary chemotherapy regimen used was Bleomycin, Epirubicin and Cisplatin (BEP) for 3-4 cycles, depending on the IGCCCG risk group. 3 good risks patients received Epirubicin and Cisplatin (EP), 5 patients received Etoposide, Ifosfamide and Cisplatin (VIP) whilst 2 patients received alternative individualised chemotherapy regimens due to comorbidities.

Surgery was performed in all patients due to residual retroperitoneal mass of $>1 \mathrm{~cm}$ on CT imaging. The operative approach was bilateral full template infrahilar RPLND via an anterior transabdominal approach. Nerve-sparing techniques were used where possible, but this was limited by significant tissue scarring in the surgical field post chemotherapy. There were no peri-operative deaths. $14 \%$ of patients required additional procedure for resection of adjacent organs intraoperatively. The overall complication rate was $23 \%(n=13)$, of which 9 were Clavien-Dindo grade I-II, 4 were Clavien-Dindo grade IIIb and one was grade IVa (table 2). Of note, $77 \%(n=10)$ of complications occurred intra-operatively and $23 \%$ $(n=3)$ occurred post-operatively. There were three cases of wound dehiscence, which occurred at day 2, day 4 and day 10 post operatively. All three cases required reoperations. One patient suffered from infarcted kidney requiring intraoperative nephrectomy and postoperative haemodialysis.

The overall histopathology analyses are presented in table 1 . Of note, $63 \%(n=36)$ were pure teratoma, $30 \%(n=17)$ were fibrosis/necrosis and $7 \%(n=4)$ were viable tumours. Of the 4 viable tumours, 3 patients had yolk sac tumours and one patient had seminoma with an embryonal component. When comparing these frequencies to those seen in historical cohorts, ${ }^{8}$ there was a statistically significant difference in the distribution of histologies (ChiSquare $\mathrm{p}=0.0187$ ). The presence of teratoma in the orchiectomy specimens is associated with the findings of teratoma in the PCRM (chi-Square $\mathrm{p}=0.0047$ ). On the contrary, the absence of teratoma is associated with the findings of fibrotic/necrotic tissue in the PCRM (chi-Square $\mathrm{p}=0.0005)$

\section{Discussion}

Despite a lack of randomised control trial evidence, there is almost universal agreement that PCRPLND should be offered to all advanced NSGCTs patients with post chemotherapy residual mass of $>1 \mathrm{~cm}$ on $\mathrm{CT}$ imaging and normal serum tumour markers. The practice is supported by retrospective surgical series, ${ }^{12,14}$ which demonstrated high proportion of residual teratoma or viable tumours at PCRPLND and the presumption that untreated teratoma or viable tumours would progress. On the other hand, it is generally accepted that observation is an appropriate management option for patients with advanced NSGCTs who achieved clinical CR (normalisation of tumour markers and resolution of all radiographic disease) following first line chemotherapy. ${ }^{15,16}$

The histopathologic evaluation of resected specimens from traditional series shows necrosis, teratoma and viable malignancy (with or without teratoma) in approximately 
$40 \%, 45 \%$ and $15 \%$ of cases respectively. ${ }^{8}$ Compared to historical data, our series show an interesting observation of: a lower proportion of viable malignancy of $7 \%(n=4)$, a higher proportion of teratoma at $63 \%(\mathrm{n}=36)$, and a lower proportion of fibrosis/necrosis at $30 \%$ $(\mathrm{n}=17)$. This is consistent with some of the more contemporary surgical series in the early 2000. ${ }^{17}$ Our series is relatively recent, capturing patients who received PCRPLND from 20102016. As the tertiary referral centre for testicular cancer in Alberta, Canada, we have a high volume of patients with testicular cancer per year. It is our policy to discuss all testicular cancer patients at a tumour board, allowing a multidisciplinary approach to patient's care and a streamlined treatment pathway. This standardizes our approach to the treatment of advanced testicular cancer in an effort to optimize outcomes. The importance of maintaining optimal chemotherapy dose intensities is emphasized, possibly resulting in lower number of viable tumours other than teratoma. Secondly, with improvement in CT imaging techniques and the availability of subspecialty radiologists, it is possible that this results in better patient selection for PCRPLND. Thirdly, we routinely perform post-chemotherapy restaging scan 8 weeks from completion of chemotherapy, allowing maximal chemotherapy effect and potentially avoiding false positive results. Lastly, men are presenting with less advanced metastatic NSGCT and the stage migration together with effective therapy may result in lower rates of viable tumors. ${ }^{9}$ On the other hand, selection bias may be contributing to the findings of high rate of teratoma and low rate of viable tumours in our series. Compared to historical series, patients who did not achieved CR post chemotherapy but have $<1 \mathrm{~cm}$ masses are currently managed with surveillance in most centres, including our centre. PCRPLND is offered only when the retroperitoneal mass grows, potentially increasing the likelihood of finding teratoma and decreasing the likelihood of finding fibrosis at PCRPLND specimens. The low rate of viable GCT in PCRPLND specimens in our series could be due to small sample size. Despite these limitations, the apparent high proportion of teratoma in our series highlights the importance of PCRPLND as an integral part of patients' management, as these tumours are often chemo-resistant and PCRPLND is associated with 10-year probability of freedom from recurrence of $80 \% .^{11}$

There have been numerous attempts made at examining clinical predictors of histology in the PCRM, in the hope to identify a subgroup of patients who can safely be observed. A number of studies have looked at the predictors of teratoma in the PCRM, and it has been consistently demonstrated that teratoma in the primary tumour is associated with higher rate of teratoma in the PCRM. ${ }^{18,19}$ In our series, $76 \%$ of patients who had teratoma in the PCRM had teratoma in the primary tumour, and we showed that the presence of teratoma in the primary tumour is associated with higher rate of teratoma in the PCRM, consistent with the literature. Other groups examined the clinical predictors for necrosis in the PCRM, including absence of teratoma in the primary tumour, normal prechemotherapy tumour markers, small residual mass and a large diminishment in mass size during chemotherapy. ${ }^{10,19}$ Our data is in line with this, where the absence of teratoma in the primary tumour was associated with presence of fibrosis/necrosis in the PCRM. However, due to the inability to reliably predict necrosis in the whole cohort of patients, these predictive models remain 
experimental and are not routinely used in clinical practice. Perhaps more importantly, patients with advanced testicular cancer can be both under or overtreated, due to a number of potential administrative and systemic issues that may affect our ability to correctly identify patients who truly require PCRPLND. In many tertiary Canadian centres, medical oncologists are often the decision maker when it comes to referring patients for PCRPLND. Meticulous review of post chemotherapy $\mathrm{CT}$ scans by a urologist familiar with the primary landing zones for testicular cancer is often not done and often reliant on radiologists. The definition of pathologic lymph node that are subcentimeter is variable and not well-defined, leading to misclassification of some patients having a CR when they likely only had a PR. Even when the urologic oncologist has a pre-determined protocol of offering PCRPLND on all PCRM of $>1 \mathrm{~cm}$ on CT scans, the system is dependent on getting the patient referred to them for consideration of surgery. Potential solutions to circumvent this systemic issue are to discuss all advanced testicular patients at a tumour board, by having a dedicated Genitourinary Radiologist to review prechemo and postchemotherapy scans, and to generate an automatic referral to Uro-oncologist for PCRPLND if the decision for referral to PCRPLND is made at tumour board.

Traditionally, RPLND is associated with high morbidity and mortality. ${ }^{20}$ With improvement in surgical expertise, anaesthetic techniques and improvement in chemotherapy resulting in smaller tumours, major complications are now less common when surgery is performed in high volume academic centres.

Many of the published surgical series used non-standardized definitions of complications, therefore, comparing complication rates cross study may be misleading. Within this limitation, the overall complication rate of $23 \%$ in our series appeared to be at the lower end of the spectrum from published surgical series, which ranges from $20 \%-35 \% .^{21,22,23}$ The mortality rate of $0 \%$ is encouraging.

Historically, increased risk of pulmonary complications was recognised in patients who received bleomycin or had bulky retroperitoneal disease. ${ }^{21}$ In our series, serious pulmonary complications were not observed. The rate of additional procedures required intraoperatively (14\%) in our series also appears lower than previously published series. ${ }^{24,25,26}$ Being a retrospective study, our study has several limitations. The identification of complications may potentially be missed if the event was not documented in the patient's clinical chart. However, the provincial electronic medical record system exists and thereby minimises the rate of undeclared complications. When there is doubt in grading the severity of a particular complication, the treating surgeon is consulted to clarify the actual event. This may cause recall or classification bias; however, the final decision about the grading of severity lies in the judgement of an independent reviewer (CLG), who used Clavien-Dindo surgical complications grading system as a reference. Our study has a relatively short followup and some longer-term complications may not be captured. Lastly, as a single surgeon derived surgical series, our results may not be generalisable to the wider audience, however, this study may provide a reference point for future study of PCRPLND in Canada.

\section{Conclusions}


PCRPLND is an integral part of the multidisciplinary management of advanced testicular patients. Our series demonstrated that the rate of viable tumour and fibrosis/necrosis in PCRM appears lower than published historical series. The high rate of teratoma in the PCRM calls for ongoing need for RPLND in the management of patients with post chemotherapy residual mass $>1 \mathrm{~cm}$. Grade 3 and 4 surgical complications are considered rare in our series. 


\section{References}

1. Cancer.ca. Toronto, ON: Canadian Cancer Society; [updated 2018 June; cited 2019 Aug 13]. cancer.ca/Canadian-Cancer-Statistics-2018-EN. Accessed August 13, 2019

2. International Germ Cell Cancer Collaborative Group. International Germ Cell Consensus Classification: A prognostic factor-based staging system for metastatic germ cell cancers. J Clin Oncol 1997;15:594-603.

3. van Dijk MR, Steyerberg EW, Habbema JD. Survival of non-seminomatous germ cell cancer patients according to the IGCC classification: An update based on metaanalysis. Eur J Cancer 2006;42:820-26.

4. Mano R, Di Natale R, Sheinfeld J. Current controversies on the role of retroperitoneal lymphadenectomy for testicular cancer. Urol Oncol. 2019;37:209-18

5. Hugen $\mathrm{CM}, \mathrm{Hu} \mathrm{B}$, Jeldres $\mathrm{C}$, et al. Utilization of retroperitoneal lymph node dissection for testicular cancer in the United States: Results from the National Cancer Database (1998-2011). Urol Oncol. 2016;34:487.

6. Wells H, Hayes MC, O'Brien T, et al. Contemporary retroperitoneal lymph node dissection (RPLND) for testis cancer in the UK - a national study. BJU Int. 2017;119:91-99.

7. Daneshmand S, Albers P, Fosså SD, et al. Contemporary management of postchemotherapy testis cancer. Eur Urol. 2012;62:867-76.

8. Nyugen CT, Stephenson AJ. Role of post chemotherapy retroperitoneal lymph node dissection in advance germ cell tumours. Hematol Oncol Clin North Am 2011;25:593604.

9. Carver BS, Shayegan B, Serio A, et al. Long-term clinical outcome after postchemotherapy retroperitoneal lymph node dissection in men with residual teratoma. J Clin Oncol. 2007;25:1033-37.

10. Vergouwe Y, Steyerberg EW, Foster RS, et al. Predicting retroperitoneal histology in postchemotherapy testicular germ cell cancer: a model update and multicentre validation with more than 1000 patients. Eur Urol. 2007;51:424-32.

11. Carver BS, Bianco FJ, Shayegan B, et al. Predicting teratoma in the retroperitoneum in men undergoing post-chemotherapy retroperitoneal lymph node dissection. J Urol 2006;176:100-04.

12. Steyerberg, EW, Keizer HJ, Habbema JD. Prediction models for the histology of residual masses after chemotherapy for metastatic testicular cancer ReHiT Study Group. Int J Cancer 1999;83:856-59.

13. Dindo D, Demartines N, Clavien PA. Classification of surgical complications: A new proposal with evaluation in a cohort of 6336 patients and results of a survey. Ann Surg 2004;240:205-13.

14. Hendry WF, Norman AR, Dearnaley DP, et al. Metastatic nonseminomatous germ cell tumours of the testis: Results of elective and salvage surgery for patients with residual retroperitoneal masses. Cancer, 2002;94:1668-76.

15. Ehrlich Y, Brames MJ, Beck SD, et al. Long term follow up of cisplatin combination chemotherapy in patients with disseminated nonseminomatous germ cell tumours: Is a post chemotherapy retroperitoneal lymph node dissection needed after complete remission? J Clin Oncol 2010;28:531-36. 
16. Kollmannsberger C, Daneshmand S, So A, et al. Management of disseminated nonseminomatous germ cell tumours with risk-based chemotherapy followed by response-guided postchemotherapy surgery. J Clin Oncol 2010;28:537-42.

17. Carver, B.S., et al. Improved clinical outcome in recent years for men with metastatic nonseminomatous germ cell tumours. J Clin Oncol 2007;25:5603-08.

18. Beck SD, Foster RS, Bihrle R et al. Teratoma in the orchiectomy specimen and volume of metastasis are predictors of retroperitoneal teratoma in post-chemotherapy nonseminomatous testis cancer. J Urol 2002;168(4 Pt 1):1402-04.

19. Albers P, Weissbach L, Krege S et al. Prediction of necrosis after chemotherapy of advanced germ cell tumours: Results of a prospective multicenter trial of the German Testicular Cancer Study Group. J Urol 2004;171:1835-38.

20. Hinman F. The operative treatment of tumours of the testicles. JAMA 1914;63:2009-15

21. Baniel J, Foster RS, Rowland RG, et al. Complications of post-chemotherapy retroperitoneal lymph node dissection. J Urol 1995;153:976-80.

22. Subramanian VS, Nguyen CT, Stephenson AJ, et al. Complications of open primary and post-chemotherapy retroperitoneal lymph node dissection for testicular cancer. Urol Oncol 2010;28:504-09.

23. Baniel J, Sella A. Complications of retroperitoneal lymph node dissection in testicular cancer: Primary and post-chemotherapy. Semin Surg Oncol 1999;17:263-67.

24. Mosharafa AA, Foster RS, Koch MO, et al. Complications of post-chemotherapy retroperitoneal lymph node dissection for testis cancer. J Urol 2004;171:1839-41.

25. Cary C, Masterson TA, Bihrle R, et al. Contemporary trends in postchemotherapy retroperitoneal lymph node dissection: Additional procedures and perioperative complications. Urol Oncol 2015;33:389

26. Considine S, Heaney R, Conroy R, et al. Post-chemotherapy retroperitoneal lymph node dissection in the management of metastatic testis cancer: The 16-year experience in an Irish setting. Ir J Med Sci 2016;185:901-07.

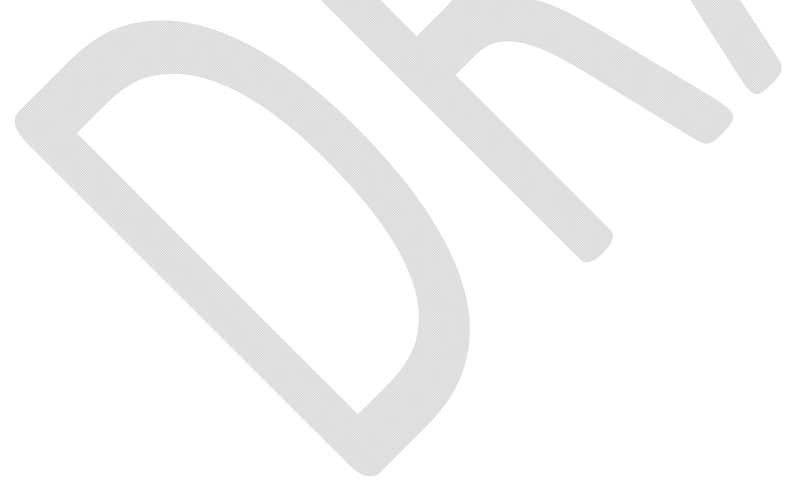


Figures and Tables

Fig. 1.

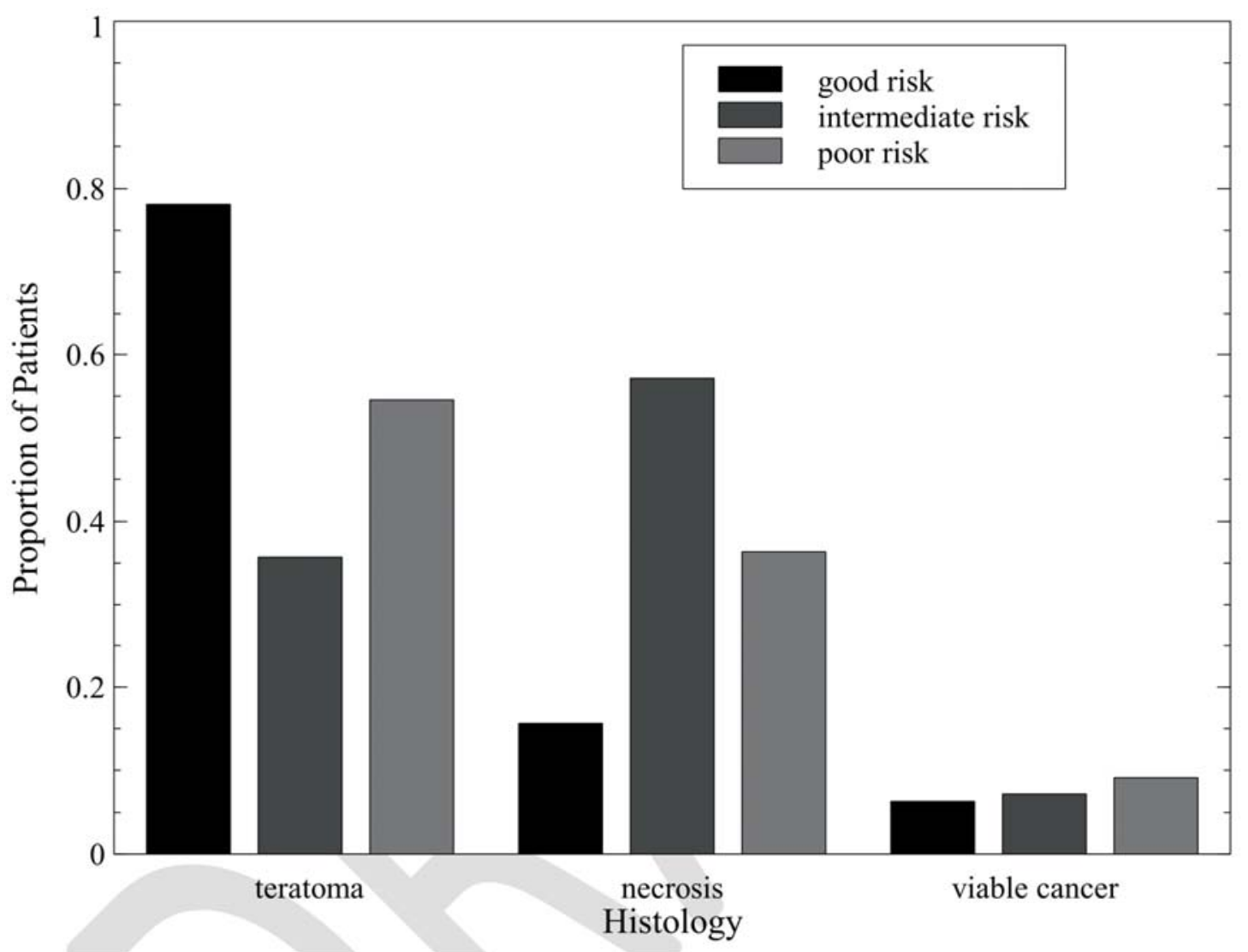




\begin{tabular}{|l|c|}
\hline \multicolumn{2}{|l|}{ Table 1. Baseline demographics and patient characteristics } \\
\hline Variable & n (\%) \\
\hline Age, median (mean) & $29(30)$ \\
\hline Clinical stage pre-chemotherapy & $1(2 \%)$ \\
\hline 1s & $5(9 \%)$ \\
\hline a & $19(33 \%)$ \\
\hline 2b & $9(16 \%)$ \\
\hline 2c & $4(7 \%)$ \\
\hline 3a & $9(16 \%)$ \\
\hline 3b & $10(18 \%)$ \\
\hline 3c & $28(49 \%)$ \\
\hline Site of primary tumor & $28(49 \%)$ \\
\hline Right & $1(2 \%)$ \\
\hline Left & \\
\hline Bilateral & $32(56 \%)$ \\
\hline Patient characteristics pre-chemotherapy & $14(25 \%)$ \\
\hline Good risk & $11(19 \%)$ \\
\hline Intermediate risk & $5.6 \pm 3.3 \mathrm{~cm}$ \\
\hline Poor risk & $0.1 \mathrm{~L}-6 \mathrm{~L}$ \\
\hline Mean size of retroperitoneal tumor \pm SD & $8(14 \%)$ \\
\hline Blood loss range & $52.4 \mathrm{months}$ \\
\hline Organs removed without complications & $36(63 \%)$ \\
\hline Median followup & $17(30 \%)$ \\
\hline Retroperitoneal histology & $4(7 \%)$ \\
\hline Teratoma & \\
\hline Necrosis & \\
\hline Viable cancer & \\
\hline SD standardeviation & \\
\hline
\end{tabular}

SD: standard deviation.

\begin{tabular}{|l|c|c|}
\hline Table 2. Descriptions and grading of surgical complications \\
\hline Grade & Complication & n \\
\hline I & Damage of IVC or aorta & 1 \\
\hline \multirow{4}{*}{ II } & Damage of renal vein & 1 \\
\cline { 2 - 3 } & Damage of IVC or aorta & 3 \\
\cline { 2 - 3 } & Thrombosis and blood transfusion & 2 \\
\cline { 2 - 3 } & Chyle leakage/infection & 1 \\
\cline { 2 - 3 } & Blood transfusion & 1 \\
\hline IIIb & Wound dehiscence (postoperative) & 3 \\
\hline IVa & $\begin{array}{c}\text { Renal infarct requiring temporary dialysis } \\
\text { and intensive care unit }\end{array}$ & 1 \\
\hline Total & & $13(22.8 \%)$ \\
\hline
\end{tabular}

IVC: inferior vena cava. 